\title{
OPERATOR IDENTITIES FOR STANDARD WEIGHTED BERGMAN SHIFT AND TOEPLITZ OPERATORS
}

\author{
ANDERS OLOFSSON AND ARON WENNMAN
}

\begin{abstract}
We prove an operator identity for the shift operator in the scale of standard weighted Bergman spaces in the unit disc. This operator identity is then applied in the context of functional calculus for the shift operator and a characterization of harmonic symbol Bergman space Toeplitz operators is obtained generalizing an earlier result by Louhichi and Olofsson. Duality arguments lead to operator inequalities and structure formulas for reproducing kernel functions which make contact with work of Richter, Shimorin, and others.
\end{abstract}

\section{INTRODUCTION}

Let $\alpha>-1$ and consider the standard weighted Bergman space $A_{\alpha}(\mathbb{D})$ of analytic functions $f$ in the unit disc $\mathbb{D}$ with finite norm

$$
\|f\|_{\alpha}^{2}=\int_{\mathbb{D}}|f(z)|^{2} \mathrm{~d} \mu_{\alpha}(z),
$$

where

$$
\mathrm{d} \mu_{\alpha}(z)=(\alpha+1)\left(1-|z|^{2}\right)^{\alpha} \mathrm{dA}(z), \quad z \in \mathbb{D} .
$$

Here $\mathrm{dA}$ is usual planar Lebesgue area measure normalized so that the unit disc has unit area. Note that the space $A_{0}(\mathbb{D})$ is the unweighted Bergman space, and that the standard Hardy space $H^{2}(\mathbb{D})$ is a natural limit case of the spaces $A_{\alpha}(\mathbb{D})$ as $\alpha \rightarrow-1$.

The norm of $A_{\alpha}(\mathbb{D})$ can also be calculated from Parseval's formula

$$
\|f\|_{\alpha}^{2}=\sum_{k \geq 0}\left|a_{k}\right|^{2} \mu_{\alpha ; k}
$$

using the power series expansion

$$
f(z)=\sum_{k \geq 0} a_{k} z^{k}, \quad z \in \mathbb{D},
$$

of $f \in A_{\alpha}(\mathbb{D})$. Here the numbers

$$
\mu_{\alpha ; k}=\int_{\mathbb{D}}|z|^{2 k} \mathrm{~d} \mu_{\alpha}(z)=1 /\left(\begin{array}{c}
k+\alpha+1 \\
k
\end{array}\right), \quad k=0,1,2, \ldots,
$$

Date: October 8, 2012.

2010 Mathematics Subject Classification. Primary: 47B35, 47B32; Secondary: 30H20, 47A60.

Key words and phrases. Bergman space, shift operator, Toeplitz operator, functional calculus, reproducing kernel function.

Research supported by GS Magnusons fond of the Royal Swedish Academy of Sciences and Lannérs stipendiefond. 
are the moments of the measure $\mathrm{d} \mu_{\alpha}$ and the generalized binomial coefficients are defined using the Gamma function (see Section 1). The scale of Hilbert spaces $A_{\alpha}(\mathbb{D})$ has been subject to much interest as documented by the monograph Hedenmalm, Korenblum and Zhu [14].

The shift operator $S_{\alpha}$ on the Bergman space $A_{\alpha}(\mathbb{D})$ is the operator defined by

$$
\left(S_{\alpha} f\right)(z)=z f(z), \quad z \in \mathbb{D}
$$

for $f \in A_{\alpha}(\mathbb{D})$. It is straightforward to see that the shift $S_{\alpha}$ is a left-invertible contraction. We show the following operator identity for the shift operator $S_{\alpha}$ :

$$
\left(S_{\alpha}^{*} S_{\alpha}\right)^{-1}=\sum_{k \geq 0}(-1)^{k}\left(\begin{array}{l}
\alpha+2 \\
k+1
\end{array}\right) S_{\alpha}^{k} S_{\alpha}^{* k}
$$

valid in the full scale $\alpha>-1$ (see Theorem 1.4). The binomial coefficient in (0.2) is $O\left(1 / k^{\alpha+3}\right.$ ) as $k \rightarrow \infty$ (see Proposition 1.2). Since $S_{\alpha}$ is a contraction, this gives that the series in (0.2) is absolutely convergent in operator norm. Also, since $S_{\alpha}$ is bounded from below the operator $\left(S_{\alpha}^{*} S_{\alpha}\right)^{-1}$ exists as a bounded linear operator on $A_{\alpha}(\mathbb{D})$.

In the special case when $n=\alpha+2$ is a positive integer formula (0.2) is known and seems first to have appeared in Olofsson [18] where it was used in a calculation of operator-valued Bergman inner functions. In the other direction, we show that an operator formula of the form (0.2) together with a pureness condition characterize the Bergman shift operator $S_{\alpha}$ up to unitary equivalence allowing for a general multiplicity (see Theorem 5.4). This last observation generalizes a recent result by Giselsson and Olofsson [12].

The left-hand side in (0.2) has the representation $\left(S_{\alpha}^{\prime}\right)^{*} S_{\alpha}^{\prime}=\left(S_{\alpha}^{*} S_{\alpha}\right)^{-1}$, where $S_{\alpha}^{\prime}=S_{\alpha}\left(S_{\alpha}^{*} S_{\alpha}\right)^{-1}$ is the so-called Cauchy dual of $S_{\alpha}$. The operator $S_{\alpha}^{\prime}$ is a weighted shift operator and acts as

$$
\left(S_{\alpha}^{\prime} f\right)(z)=\sum_{k \geq 1} \frac{\mu_{\alpha ; k-1}}{\mu_{\alpha ; k}} a_{k-1} z^{k}, \quad z \in \mathbb{D}
$$

on functions $f \in A_{\alpha}(\mathbb{D})$ given by (0.1). Formula (0.3) follows by straightforward calculation (see Lemma 1.1).

Motivated by $(0.2)$ we turn to a study of bounded linear operators $T \in \mathcal{L}\left(A_{\alpha}(\mathbb{D})\right)$ on $A_{\alpha}(\mathbb{D})$ satisfying the operator identity

$$
\left(S_{\alpha}^{\prime}\right)^{*} T S_{\alpha}^{\prime}=\sum_{k \geq 0}(-1)^{k}\left(\begin{array}{c}
\alpha+2 \\
k+1
\end{array}\right) S_{\alpha}^{k} T S_{\alpha}^{* k} .
$$

Recall that the sum in (0.4) is absolutely convergent in operator norm by decay of binomial coefficients (see Proposition 1.2). We provide two descriptions of the operators $T \in \mathcal{L}\left(A_{\alpha}(\mathbb{D})\right)$ satisfying (0.4): First as operator integrals

$$
T=\int_{\mathbb{T}} f\left(e^{i \theta}\right) \mathrm{d} \omega_{S_{\alpha}}\left(e^{i \theta}\right)
$$

of functions $f \in L^{\infty}(\mathbb{T})$ with respect to the Bergman shift operator $S_{\alpha}$ (see Theorem 2.5) and, second, as Toeplitz operators $T=T_{h}$ on $A_{\alpha}(\mathbb{D})$ with bounded harmonic symbols $h$ (see Theorem 3.3). Here $\mathbb{T}=\partial \mathbb{D}$ is the unit circle and the Toeplitz operator on $A_{\alpha}(\mathbb{D})$ with bounded harmonic symbol $h$ is the operator $T_{h}$ 
defined by

$$
\left(T_{h} g\right)(z)=\int_{\mathbb{D}} \frac{1}{(1-\bar{\zeta} z)^{\alpha+2}} h(\zeta) g(\zeta) \mathrm{d} \mu_{\alpha}(\zeta), \quad z \in \mathbb{D},
$$

for $g \in A_{\alpha}(\mathbb{D})$. The operator measure $\mathrm{d} \omega_{S_{\alpha}}$ can be thought of as the compression to $A_{\alpha}(\mathbb{D})$ of the spectral measure for a unitary dilation of the operator $S_{\alpha}$. The relation between the symbols $f$ and $h$ is that $h=P[f]$ is the Poisson integral of $f$ (see Theorem 3.2).

In the special case when $n=\alpha+2$ is a positive integer the above characterizations of operators satisfying (0.4) are from Louhichi and Olofsson [15]. The novelty here is the generalization of these results to the full parameter scale $\alpha>-1$. Our proofs build on developments from [15] using the new ingredient (0.2). As a historical background we mention the classical paper Brown and Halmos [8] which has inspired much research on Toeplitz operators. Examples of more recent progress on Toeplitz operators in the Bergman space context are Axler and Cucucković [7], Ahern and Čučković [2] and Ahern [1].

In the restricted parameter range $-1<\alpha \leq 0$ we show by a duality argument that the shift operator $S_{\alpha}$ satisfies the inequality

$$
\left\|f+\sum_{k \geq 1} C_{\alpha ; k} S_{\alpha}^{k} f_{k}\right\|_{\alpha}^{2} \leq(\alpha+2)\left(\left\|S_{\alpha} f\right\|_{\alpha}^{2}+\sum_{k \geq 1}\left\|f_{k}\right\|_{\alpha}^{2}\right)
$$

for $f \in A_{\alpha}(\mathbb{D})$ and $\left\{f_{k}\right\}_{k \geq 1} \in \ell^{2}\left(A_{\alpha}(\mathbb{D})\right)$ finitely supported, where the $C_{\alpha ; k}$ 's are arbitrary complex numbers such that

$$
\left|C_{\alpha ; k}\right|^{2}=(-1)^{k+1}\left(\begin{array}{c}
\alpha+2 \\
k+1
\end{array}\right)
$$

for $k=1,2,3, \ldots$ (see Corollary 4.2). An interesting feature of inequality (0.5) is that it is stable with respect to the process of passing to restrictions to shift invariant subspaces. It should be noticed that (0.5) for $\alpha=0$ gives the inequality

$$
\left\|f+S_{0} g\right\|_{0}^{2} \leq 2\left(\left\|S_{0} f\right\|_{0}^{2}+\|g\|_{0}^{2}\right), \quad f, g \in A_{0}(\mathbb{D})
$$

from Hedenmalm, Jakobsson and Shimorin [13, Proposition 6.4]. A further analysis of inequalities derived from (0.5) leads to structure formulas for (normalized) reproducing kernel functions similar to what has previously been obtained by Shimorin [21] and McCullough and Richter [16] (see Theorem 5.5). See [3, 9, 17, 22, 23] for related results. A main inspiration behind these developments have been the ground breaking paper Aleman, Richter and Sundberg [4].

\section{THE OPERATOR IDENTITY}

In this section we shall prove the operator identity (0.2). For the purpose of more generality we shall consider vector-valued versions of the spaces $A_{\alpha}(\mathbb{D})$. Let $\alpha>-1$ and let $\mathcal{E}$ be a Hilbert space. We denote by $A_{\alpha}(\mathcal{E})$ the space of all $\mathcal{E}$-valued analytic functions

$$
f(z)=\sum_{k \geq 0} a_{k} z^{k}, \quad z \in \mathbb{D}
$$

with finite norm

$$
\|f\|_{\alpha}^{2}=\sum_{k \geq 0}\left\|a_{k}\right\|^{2} \mu_{\alpha ; k}<+\infty
$$


It it straightforward to check that the space $A_{\alpha}(\mathcal{E})$ is a Hilbert space of $\mathcal{E}$-valued analytic functions in $\mathbb{D}$ with reproducing kernel function

$$
K_{\alpha}(z, \zeta)=\frac{1}{(1-\bar{\zeta} z)^{\alpha+2}} I_{\mathcal{E}}=\left(\sum_{k \geq 0}\left(\begin{array}{c}
k+\alpha+1 \\
k
\end{array}\right) \bar{\zeta}^{k} z^{k}\right) I_{\mathcal{E}}, \quad(z, \zeta) \in \mathbb{D}^{2}
$$

where $I_{\mathcal{E}}$ is the identity operator in $\mathcal{L}(\mathcal{E})$. A standard reference for Bergman spaces in $\mathbb{D}$ is Hedenmalm, Korenblum and Zhu [14]; see also Duren and Schuster [11].

The shift operator acts as

$$
S_{\alpha} f(z)=\sum_{k \geq 1} a_{k-1} z^{k}, \quad z \in \mathbb{D}
$$

on functions $f \in A_{\alpha}(\mathcal{E})$ given by (1.1). The adjoint shift $S_{\alpha}^{*}$ acts as

$$
S_{\alpha}^{*} f(z)=\sum_{k \geq 0} \frac{\mu_{\alpha ; k+1}}{\mu_{\alpha ; k}} a_{k+1} z^{k}, \quad z \in \mathbb{D},
$$

on functions $f \in A_{\alpha}(\mathcal{E})$ given by (1.1). Formula (1.2) is easily checked by straightforward calculation. Let us calculate the action of the operator $\left(S_{\alpha}^{*} S_{\alpha}\right)^{-1}$.

Lemma 1.1. Let $\alpha>-1$. Then

$$
\left(S_{\alpha}^{*} S_{\alpha}\right)^{-1} f(z)=\sum_{k \geq 0} \frac{\mu_{\alpha ; k}}{\mu_{\alpha ; k+1}} a_{k} z^{k}, \quad z \in \mathbb{D}
$$

for $f \in A_{\alpha}(\mathcal{E})$ given by (1.1).

Proof. By the action of $S_{\alpha}$ we have

$$
\left\langle S_{\alpha}^{*} S_{\alpha} f, f\right\rangle_{\alpha}=\left\|S_{\alpha} f\right\|_{\alpha}^{2}=\sum_{k \geq 1}\left\|a_{k-1}\right\|^{2} \mu_{\alpha ; k}=\sum_{k \geq 0}\left\|a_{k}\right\|^{2} \mu_{\alpha ; k+1},
$$

which by polarization gives

$$
S_{\alpha}^{*} S_{\alpha} f(z)=\sum_{k \geq 0} \frac{\mu_{\alpha ; k+1}}{\mu_{\alpha ; k}} a_{k} z^{k}, \quad z \in \mathbb{D},
$$

for $f \in A_{\alpha}(\mathcal{E})$ given by (1.1). Passing to the inverse $\left(S_{\alpha}^{*} S_{\alpha}\right)^{-1}$ the conclusion of the lemma follows.

The reciprocal of the Bergman kernel function $K_{\alpha}$ is essentially the binomial series

$$
(1-z)^{\alpha+2}=\sum_{k \geq 0}(-1)^{k}\left(\begin{array}{c}
\alpha+2 \\
k
\end{array}\right) z^{k}, \quad z \in \mathbb{D},
$$

where the binomial coefficients are interpreted in the generalized sense

$$
\left(\begin{array}{l}
\beta \\
k
\end{array}\right)=\frac{\Gamma(\beta+1)}{k ! \Gamma(\beta-k+1)}=\frac{1}{k !} \prod_{j=0}^{k-1}(\beta-j)
$$

for $k=0,1,2, \ldots$ using the Gamma function $\Gamma$.

We shall need some properties of the coefficients in (1.3). 
Proposition 1.2. Let $\alpha>-1$. Then $(-1)^{k}\left(\begin{array}{c}\alpha+2 \\ k\end{array}\right) \geq 0$ for $k>\alpha+2$ if $2 m-1 \leq$ $\alpha \leq 2 m$ for some integer $m \geq 0$. Similarly $(-1)^{k}\left(\begin{array}{c}\alpha+2 \\ k\end{array}\right) \leq 0$ for $k>\alpha+2$ if $2 m \leq \alpha \leq 2 m+1$ for some integer $m \geq 0$. Furthermore, the coefficients in (1.3) have the growth property that

$$
\lim _{k \rightarrow \infty}(-1)^{k}\left(\begin{array}{c}
\alpha+2 \\
k
\end{array}\right) k^{\alpha+3}=\frac{\Gamma(\alpha+3) \sin (\pi(\alpha+1))}{\pi},
$$

where $\Gamma$ is the Gamma function.

Proof. Writing out the generalized binomial coefficient we have

$$
\left(\begin{array}{c}
\alpha+2 \\
k
\end{array}\right)=\frac{\Gamma(\alpha+3) \Gamma(k-\alpha-2)}{k ! \Gamma(k-\alpha-2) \Gamma(1-(k-\alpha-2))}
$$

for $k=0,1, \ldots$ Using the reflection formula

$$
\Gamma(z) \Gamma(1-z)=\frac{\pi}{\sin \pi z}
$$

for the Gamma function (see Artin [6, formula (4.5)]) we obtain

$$
\left(\begin{array}{c}
\alpha+2 \\
k
\end{array}\right)=\Gamma(\alpha+3) \frac{\sin \pi(k-\alpha-2)}{\pi} \frac{\Gamma(k-\alpha-2)}{k !},
$$

which by the addition formula for the sine simplifies to

$$
\left(\begin{array}{c}
\alpha+2 \\
k
\end{array}\right)=(-1)^{k} \Gamma(\alpha+3) \frac{\sin \pi(\alpha+1)}{\pi} \frac{\Gamma(k-\alpha-2)}{k !}
$$

for $k=0,1, \ldots$ Analyzing the sign of the individual factors in (1.4) we obtain the conclusions about the sign of $(-1)^{k}\left(\begin{array}{c}\alpha+2 \\ k\end{array}\right)$. A calculation using Stirling's formula

$$
\lim _{x \rightarrow+\infty} \frac{\Gamma(x+1)}{(x / e)^{x} \sqrt{2 \pi x}}=1
$$

(see $[19, \S 8.22])$ gives the asymptotic behavior of the numbers $(-1)^{k}\left(\begin{array}{c}\alpha+2 \\ k\end{array}\right)$.

The proof of identity (0.2) uses the following lemma on binomial coefficients.

Lemma 1.3. Let $\mu_{\alpha ; k}=1 /(\underset{k}{k+\alpha+1})$ for $\alpha>-1$ and $k \geq 0$. Then

$$
\sum_{k=0}^{j}(-1)^{k}\left(\begin{array}{l}
\alpha+2 \\
k+1
\end{array}\right) \frac{1}{\mu_{\alpha ; j-k}}=\frac{1}{\mu_{\alpha ; j+1}}
$$

for $j \geq 0$.

Proof. A calculation using the binomial series (1.3) gives that

$$
\sum_{k \geq 0}(-1)^{k}\left(\begin{array}{l}
\alpha+2 \\
k+1
\end{array}\right) z^{k}=\frac{1-(1-z)^{\alpha+2}}{z}, \quad z \in \mathbb{D} .
$$

Observe that the sum in the lemma is the $j$-th Taylor coefficient for the function

$$
f(z)=\frac{1-(1-z)^{\alpha+2}}{z} \frac{1}{(1-z)^{\alpha+2}}, \quad z \in \mathbb{D} .
$$

Calculating the Taylor series expansion for $f$ we have

$$
f(z)=\frac{1}{z}\left(\frac{1}{(1-z)^{\alpha+2}}-1\right)=\sum_{j \geq 0} \frac{1}{\mu_{\alpha ; j+1}} z^{j}, \quad z \in \mathbb{D} .
$$

An identification of coefficients now yields the conclusion of the lemma. 
We are now ready for the proof of $(0.2)$.

Theorem 1.4. Let $\alpha>-1$ and let $\mathcal{E}$ be a Hilbert space. Then the shift operator $S_{\alpha}$ on $A_{\alpha}(\mathcal{E})$ satisfies the operator identity

$$
\left(S_{\alpha}^{*} S_{\alpha}\right)^{-1}=\sum_{k \geq 0}(-1)^{k}\left(\begin{array}{c}
\alpha+2 \\
k+1
\end{array}\right) S_{\alpha}^{k} S_{\alpha}^{* k} .
$$

Proof. Observe that the sum in (0.2) is absolutely convergent in operator norm as follows by Proposition 1.2. Let $f \in A_{\alpha}(\mathcal{E})$ be a function of the form (1.1). By (1.2) we have that

$$
\left\|S_{\alpha}^{* k} f\right\|_{\alpha}^{2}=\sum_{j \geq 0} \frac{\mu_{\alpha ; j+k}^{2}}{\mu_{\alpha ; j}}\left\|a_{j+k}\right\|^{2}
$$

for $k \geq 0$. We calculate that

$$
\begin{aligned}
\sum_{k \geq 0}(-1)^{k}\left(\begin{array}{l}
\alpha+2 \\
k+1
\end{array}\right)\left\|S_{\alpha}^{* k} f\right\|_{\alpha}^{2} & =\sum_{k \geq 0} \sum_{j \geq 0}(-1)^{k}\left(\begin{array}{l}
\alpha+2 \\
k+1
\end{array}\right) \frac{\mu_{\alpha ; j+k}^{2}}{\mu_{\alpha ; j}}\left\|a_{j+k}\right\|^{2} \\
& =\sum_{l \geq 0}\left(\sum_{k=0}^{l}(-1)^{k}\left(\begin{array}{l}
\alpha+2 \\
k+1
\end{array}\right) \frac{1}{\mu_{\alpha ; l-k}}\right) \mu_{\alpha ; l}^{2}\left\|a_{l}\right\|^{2},
\end{aligned}
$$

where the last equality follows by a change of order of summation. Now use Lemma 1.3 to conclude that

$$
\sum_{k \geq 0}(-1)^{k}\left(\begin{array}{l}
\alpha+2 \\
k+1
\end{array}\right)\left\|S_{\alpha}^{* k} f\right\|_{\alpha}^{2}=\sum_{j \geq 0} \frac{\mu_{\alpha ; j}^{2}}{\mu_{\alpha ; j+1}}\left\|a_{j}\right\|^{2} .
$$

By Lemma 1.1 this last equality yields that

$$
\sum_{k \geq 0}(-1)^{k}\left(\begin{array}{l}
\alpha+2 \\
k+1
\end{array}\right)\left\|S_{\alpha}^{* k} f\right\|_{\alpha}^{2}=\left\langle\left(S_{\alpha}^{*} S_{\alpha}\right)^{-1} f, f\right\rangle_{\alpha}
$$

for $f \in A_{\alpha}(\mathcal{E})$. The conclusion of the theorem now follows by a polarization argument.

We mention that for $n=\alpha+2$ positive integer the result of Theorem 1.4 originates from [18, Section 1] where it was used in a calculation of operator-valued Bergman inner functions.

\section{Functional CAlCulus}

In this section and the next we shall study bounded linear operators $T$ in $\mathcal{L}\left(A_{\alpha}(\mathbb{D})\right)$ satisfying the operator identity

$$
\left(S_{\alpha}^{\prime}\right)^{*} T S_{\alpha}^{\prime}=\sum_{k \geq 0}(-1)^{k}\left(\begin{array}{c}
\alpha+2 \\
k+1
\end{array}\right) S_{\alpha}^{k} T S_{\alpha}^{* k},
$$

where $S_{\alpha}^{\prime}=S_{\alpha}\left(S_{\alpha}^{*} S_{\alpha}\right)^{-1}$ is the so-called Cauchy dual of $S_{\alpha}$. Observe that the sum in (0.4) is absolutely convergent in operator norm by Proposition 1.2 since $S_{\alpha}$ is a contraction. In this section we focus on the description of this class of operators in terms of functional calculus for the shift operator $S_{\alpha}$.

For a Hilbert space operator $T \in \mathcal{L}(\mathcal{H})$ and integer $k \in \mathbb{Z}$ we use the notation

$$
T(k)= \begin{cases}T^{k} & \text { for } k \geq 0 \\ T^{*|k|} & \text { for } k<0\end{cases}
$$


which is standard in dilation theory (see [24, Chapter I]).

Proposition 2.1. Let $\alpha>-1$ and $n \in \mathbb{Z}$. Then the operator $T=S_{\alpha}(n)$ satisfies (0.4).

Proof. For $n \geq 0$ we have

$$
\left(S_{\alpha}^{\prime}\right)^{*} S_{\alpha}(n) S_{\alpha}^{\prime}=\left(S_{\alpha}^{*} S_{\alpha}\right)^{-1} S_{\alpha}^{*} S_{\alpha}^{n} S_{\alpha}\left(S_{\alpha}^{*} S_{\alpha}\right)^{-1}=S_{\alpha}^{n}\left(S_{\alpha}^{*} S_{\alpha}\right)^{-1},
$$

and using Theorem 1.4 we obtain that

$$
\left(S_{\alpha}^{\prime}\right)^{*} S_{\alpha}(n) S_{\alpha}^{\prime}=S_{\alpha}^{n}\left(\sum_{k \geq 0}(-1)^{k}\left(\begin{array}{l}
\alpha+2 \\
k+1
\end{array}\right) S_{\alpha}^{k} S_{\alpha}^{* k}\right)=\sum_{k \geq 0}(-1)^{k}\left(\begin{array}{l}
\alpha+2 \\
k+1
\end{array}\right) S_{\alpha}^{k} S_{\alpha}(n) S_{\alpha}^{* k}
$$

This gives the result for $n \geq 0$. For $n<0$ the result follows by a passage to adjoints.

Notice that for $n=0$ the result of Proposition 2.1 is merely a restatement of Theorem 1.4.

The function space $A_{\alpha}(\mathbb{D})$ is naturally equipped by a unitary group of translation operators $\tau: e^{i \theta} \mapsto \tau_{e^{i \theta}}$, where

$$
\tau_{e^{i \theta}} f(z)=f\left(z e^{-i \theta}\right), \quad z \in \mathbb{D},
$$

for $f \in A_{\alpha}(\mathbb{D})$ and $e^{i \theta} \in \mathbb{T}$. The map $\tau: \mathbb{T} \ni e^{i \theta} \mapsto \tau_{e^{i \theta}} \in \mathcal{L}\left(A_{\alpha}(\mathbb{D})\right)$ is continuous in the strong operator topology. We say that an operator $T \in \mathcal{L}\left(A_{\alpha}(\mathbb{D})\right)$ is homogeneous of degree $k \in \mathbb{Z}$ with respect to the group of translations if it has the property that

$$
\tau_{e^{i \theta}} T \tau_{e^{-i \theta}}=e^{-i k \theta} T
$$

for $e^{i \theta} \in \mathbb{T}$. Observe that an operator of homogeneity 0 is a Fourier multiplier.

For a general operator $T \in \mathcal{L}\left(A_{\alpha}(\mathbb{D})\right)$ we shall consider its $k$-th homogeneous part given by

$$
T_{k} f=\frac{1}{2 \pi} \int_{\mathbb{T}} e^{-i k \theta} \tau_{e^{-i \theta}} T \tau_{e^{i \theta}} f \mathrm{~d} \theta, \quad f \in A_{\alpha}(\mathbb{D}),
$$

for $k \in \mathbb{Z}$, where the integral is interpreted as a standard $A_{\alpha}(\mathbb{D})$-valued integral of a continuous function. A change of variables in (2.2) shows that $T_{k}$ is homogeneous of degree $k$ in the above sense. Standard harmonic analysis arguments show that a general operator $T \in \mathcal{L}\left(A_{\alpha}(\mathbb{D})\right)$ can be reconstructed from its homogeneous parts as a Cesàro limit

$$
T=\lim _{N \rightarrow \infty} \sum_{|k| \leq N}\left(1-\frac{|k|}{N+1}\right) T_{k}
$$

in the strong operator topology in $\mathcal{L}\left(A_{\alpha}(\mathbb{D})\right)$ (see [15, Section 2] for details).

In the upcoming lemmas we consider operators $T \in \mathcal{L}\left(A_{\alpha}(\mathbb{D})\right)$ satisfying $(0.4)$ and an additional homogeneity constraint.

Lemma 2.2. Assume that $T \in \mathcal{L}\left(A_{\alpha}(\mathbb{D})\right)$ is homogeneous of degree 0 and that $T$ satisfies $(0.4)$. Then $T$ is a constant multiple of the identity operator $I$ on $A_{\alpha}(\mathbb{D})$.

Proof. The operator $T$ being homogeneous of degree 0 amounts to saying that $T$ is a Fourier multiplier in the sense that

$$
(T f)(z)=\sum_{j \geq 0} t_{j} a_{j} z^{j}, \quad z \in \mathbb{D},
$$


for $f \in A_{\alpha}(\mathbb{D})$ given by $(0.1)$, where $\left\{t_{j}\right\}_{j=0}^{\infty}$ is a sequence of complex numbers. Boundedness of $T$ corresponds to the multiplier sequence $\left\{t_{j}\right\}_{j=0}^{\infty}$ being bounded. The relation (0.4) means that

$$
\left\langle T S_{\alpha}^{\prime} f, S_{\alpha}^{\prime} f\right\rangle_{\alpha}=\sum_{k \geq 0}(-1)^{k}\left(\begin{array}{c}
\alpha+2 \\
k+1
\end{array}\right)\left\langle T S_{\alpha}^{* k} f, S_{\alpha}^{* k} f\right\rangle_{\alpha}
$$

for $f \in A_{\alpha}(\mathbb{D})$.

We shall next calculate the scalar products in (2.4). By (0.3) we have that

$$
\left\langle T S_{\alpha}^{\prime} f, S_{\alpha}^{\prime} f\right\rangle_{\alpha}=\sum_{j \geq 0} t_{j+1} \frac{\mu_{\alpha ; j}^{2}}{\mu_{\alpha ; j+1}}\left|a_{j}\right|^{2}
$$

for $f \in A_{\alpha}(\mathbb{D})$ given by $(0.1)$. By (1.2) we have that

$$
\begin{array}{r}
\sum_{k \geq 0}(-1)^{k}\left(\begin{array}{l}
\alpha+2 \\
k+1
\end{array}\right)\left\langle T S_{\alpha}^{* k} f, S_{\alpha}^{* k} f\right\rangle_{\alpha}=\sum_{k \geq 0} \sum_{j \geq 0}(-1)^{k}\left(\begin{array}{l}
\alpha+2 \\
k+1
\end{array}\right) t_{j} \frac{\mu_{\alpha ; j+k}^{2}}{\mu_{\alpha ; j}}\left|a_{j+k}\right|^{2} \\
=\sum_{j \geq 0}\left(\sum_{k=0}^{j}(-1)^{k}\left(\begin{array}{l}
\alpha+2 \\
k+1
\end{array}\right) \frac{t_{j-k}}{\mu_{\alpha ; j-k}}\right)\left|a_{j}\right|^{2} \mu_{\alpha ; j}^{2}
\end{array}
$$

for $f \in A_{\alpha}(\mathbb{D})$ given by $(0.1)$, where the last equality follows by a change of order of summation.

Using equations $(2.4),(2.5)$ and $(2.6)$ and varying $f \in A_{\alpha}(\mathbb{D})$ of the form $(0.1)$, we conclude that

$$
\frac{t_{j+1}}{\mu_{\alpha ; j+1}}=\sum_{k=0}^{j}(-1)^{k}\left(\begin{array}{c}
\alpha+2 \\
k+1
\end{array}\right) \frac{t_{j-k}}{\mu_{\alpha ; j-k}}
$$

for $j \geq 0$. Recall Lemma 1.3. By (2.7) we conclude that $t_{j}=t_{0}$ for $j \geq 1$. This gives the conclusion that $T=t_{0} I$.

The following lemma allows for reduction to homogeneity 0 .

Lemma 2.3. Let $T \in \mathcal{L}\left(A_{\alpha}(\mathbb{D})\right)$ be homogeneous of degree $k \geq 0$. Then $T=$ $S_{\alpha}^{k} L_{\alpha}^{k} T$, where $L_{\alpha}=\left(S_{\alpha}^{*} S_{\alpha}\right)^{-1} S_{\alpha}^{*}$ is the Moore-Penrose left-inverse of $S_{\alpha}$.

Proof. It is straightforward to check that the range of $T$ is contained in the range of $S_{\alpha}^{k}$ (see [15, Lemma 3.2]). This gives the factorization $T=S_{\alpha}^{k} L_{\alpha}^{k} T$.

We can now describe a general operator $T \in \mathcal{L}\left(A_{\alpha}(\mathbb{D})\right)$ satisfying (0.4). Recall the notation (2.1).

Theorem 2.4. Let $T \in \mathcal{L}\left(A_{\alpha}(\mathbb{D})\right)$ be a bounded linear operator. Then $T$ satisfies (0.4) if and only if

$$
T_{k}=c_{k} S_{\alpha}(k), \quad k \in \mathbb{Z},
$$

for some sequence $\left\{c_{k}\right\}_{k=-\infty}^{\infty}$ of complex numbers, where $T_{k}$ is the $k$-th homogeneous part of $T$.

Proof. We consider first the if-part. By Proposition 2.1 every homogeneous part $T_{k}=c_{k} S_{\alpha}(k)$ of $T$ satisfies (0.4). By the approximation property (2.3) we conclude that the operator $T$ satisfies $(0.4)$. 
Assume next that $T \in \mathcal{L}\left(A_{\alpha}(\mathbb{D})\right)$ satisfies $(0.4)$. Since the operators $S_{\alpha}$ and $S_{\alpha}^{\prime}$ are both homogeneous of degree 1 , we have that

$$
\begin{aligned}
\left(S_{\alpha}^{\prime}\right)^{*} \tau_{e^{-i \theta}} T \tau_{e^{i \theta}} S_{\alpha}^{\prime} & =\tau_{e^{-i \theta}}\left(S_{\alpha}^{\prime}\right)^{*} T S_{\alpha}^{\prime} \tau_{e^{i \theta}}=\tau_{e^{-i \theta}}\left(\sum_{n \geq 0}(-1)^{n}\left(\begin{array}{c}
\alpha+2 \\
n+1
\end{array}\right) S_{\alpha}^{n} T S_{\alpha}^{* n}\right) \tau_{e^{i \theta}} \\
& =\sum_{n \geq 0}(-1)^{n}\left(\begin{array}{l}
\alpha+2 \\
n+1
\end{array}\right) S_{\alpha}^{n} \tau_{e^{-i \theta}} T \tau_{e^{i \theta}} S_{\alpha}^{* n}
\end{aligned}
$$

for $e^{i \theta} \in \mathbb{T}$. Now using (2.2) we calculate that

$$
\begin{aligned}
\left(S_{\alpha}^{\prime}\right)^{*} T_{k} S_{\alpha}^{\prime} f & =\frac{1}{2 \pi} \int_{\mathbb{T}} e^{-i k \theta}\left(S_{\alpha}^{\prime}\right)^{*} \tau_{e^{-i \theta}} T \tau_{e^{i \theta}} S_{\alpha}^{\prime} f \mathrm{~d} \theta \\
& =\sum_{n \geq 0}(-1)^{n}\left(\begin{array}{l}
\alpha+2 \\
n+1
\end{array}\right) \frac{1}{2 \pi} \int_{\mathbb{T}} e^{-i k \theta} S_{\alpha}^{n} \tau_{e^{-i \theta}} T \tau_{e^{i \theta}} S_{\alpha}^{* n} f \mathrm{~d} \theta \\
& =\sum_{n \geq 0}(-1)^{n}\left(\begin{array}{l}
\alpha+2 \\
n+1
\end{array}\right) S_{\alpha}^{n} T_{k} S_{\alpha}^{* n} f
\end{aligned}
$$

for $f \in A_{\alpha}(\mathbb{D})$. This shows that every $k$-th homogeneous part $T_{k}$ of $T$ satisfies $(0.4)$.

Let now $k \geq 0$ and consider the $k$-th homogeneous part $T_{k}$. We shall show that $T_{k}=c_{k} S_{\alpha}^{k}$ for some constant $c_{k} \in \mathbb{C}$. By Lemma 2.3 we have the factorization $T_{k}=S_{\alpha}^{k} L_{\alpha}^{k} T_{k}$. Next we show that $L_{\alpha}^{k} T_{k}$ satisfies $(0.4)$. Since $\left(S_{\alpha}^{\prime}\right)^{*}=L_{\alpha}$, we have

$$
\left(S_{\alpha}^{\prime}\right)^{*} L_{\alpha}^{k} T_{k} S_{\alpha}^{\prime}=L_{\alpha}^{k}\left(S_{\alpha}^{\prime}\right)^{*} T_{k} S_{\alpha}^{\prime}=L_{\alpha}^{k}\left(\sum_{n \geq 0}(-1)^{n}\left(\begin{array}{c}
\alpha+2 \\
n+1
\end{array}\right) S_{\alpha}^{n} T_{k} S_{\alpha}^{* n}\right),
$$

and using the factorization $T_{k}=S_{\alpha}^{k} L_{\alpha}^{k} T_{k}$ we calculate that

$$
\begin{aligned}
\left(S_{\alpha}^{\prime}\right)^{*} L_{\alpha}^{k} T_{k} S_{\alpha}^{\prime} & =\sum_{n \geq 0}(-1)^{n}\left(\begin{array}{c}
\alpha+2 \\
n+1
\end{array}\right) L_{\alpha}^{k} S_{\alpha}^{n+k} L_{\alpha}^{k} T_{k} S_{\alpha}^{* n} \\
& =\sum_{n \geq 0}(-1)^{n}\left(\begin{array}{c}
\alpha+2 \\
n+1
\end{array}\right) S_{\alpha}^{n} L_{\alpha}^{k} T_{k} S_{\alpha}^{* n} .
\end{aligned}
$$

Since the operator $L_{\alpha}$ is homogeneous of degree -1 , the operator $L_{\alpha}^{k} T_{k}$ has homogeneity 0. By Lemma 2.2 we conclude that $L_{\alpha}^{k} T_{k}=c_{k} I$ for some $c_{k} \in \mathbb{C}$. Solving for $T_{k}$ we get $T_{k}=c_{k} S_{\alpha}^{k}$ for $k \geq 0$.

Assume now that $k<0$. Then $T_{k}^{*}$ is homogeneous of degree $|k|$ and satisfies (0.4). By the result of the previous paragraph we have $T_{k}^{*}=\bar{c}_{k} S_{\alpha}^{|k|}$ for some $c_{k} \in \mathbb{C}$, and a passage to adjoints yields $T_{k}=c_{k} S_{\alpha}^{*|k|}$. This completes the proof of the theorem.

Let $T \in \mathcal{L}(\mathcal{H})$ be a Hilbert space contraction operator, and recall the notation (2.1). The existence of a unitary dilation of $T$ yields existence of a positive operator measure $d \omega_{T}$ on $\mathbb{T}$ such that

$$
\int_{\mathbb{T}} e^{i k \theta} \mathrm{d} \omega_{T}\left(e^{i \theta}\right)=T(k), \quad k \in \mathbb{Z}
$$

This last property determines $\mathrm{d} \omega_{T}$ uniquely within the class of positive operator measures justifying the notation $\mathrm{d} \omega_{T}$. A classical result of Sz.-Nagy and Foias gives that $\mathrm{d} \omega_{T}$ is absolutely continuous with respect to Lebesgue measure on $\mathbb{T}$ if 
$T \in \mathcal{L}(\mathcal{H})$ is a completely non-unitary contraction (see [24, Theorem II.6.4]). In the case of a shift operator $S$ the absolute continuity of $\mathrm{d} \omega_{S}$ is more easily verified (see for instance [15]). For $\mathrm{d} \omega_{T}$ absolutely continuous the functional calculus

$$
L^{\infty}(\mathbb{T}) \ni f \mapsto \int_{\mathbb{T}} f\left(e^{i \theta}\right) \mathrm{d} \omega_{T}\left(e^{i \theta}\right) \in \mathcal{L}(\mathcal{H})
$$

has the approximation property that

$$
\int_{\mathbb{T}} f\left(e^{i \theta}\right) \mathrm{d} \omega_{T}\left(e^{i \theta}\right)=\lim _{N \rightarrow \infty} \sum_{|k| \leq N}\left(1-\frac{|k|}{N+1}\right) \hat{f}(k) T(k)
$$

in the strong operator topology in $\mathcal{L}(\mathcal{H})$ for every $f \in L^{\infty}(\mathbb{T})$, where

$$
\hat{f}(k)=\frac{1}{2 \pi} \int_{\mathbb{T}} e^{-i k \theta} f\left(e^{i \theta}\right) \mathrm{d} \theta, \quad k \in \mathbb{Z},
$$

are the Fourier coefficients of $f$ (see for instance [15, Section 4]).

Next we describe the operators $T \in \mathcal{L}\left(A_{\alpha}(\mathbb{D})\right)$ satisfying identity (0.4) using functional calculus integrals.

Theorem 2.5. Let $\alpha>-1$ and $T \in \mathcal{L}\left(A_{\alpha}(\mathbb{D})\right)$. Then $T$ satisfies $(0.4)$ if and only if $T$ has the form of an operator integral

$$
T=\int_{\mathbb{T}} f\left(e^{i \theta}\right) \mathrm{d} \omega_{S_{\alpha}}\left(e^{i \theta}\right) \quad \text { in } \mathcal{L}\left(A_{\alpha}(\mathbb{D})\right)
$$

for some function $f \in L^{\infty}(\mathbb{T})$. Furthermore, we have that $\|T\|=\|f\|_{\infty}$.

Proof. Assume first that $T$ satisfies (0.4). By Theorem 2.4 and (2.3) we have that

$$
T=\lim _{N \rightarrow \infty} \sum_{|k| \leq N}\left(1-\frac{|k|}{N+1}\right) c_{k} S_{\alpha}(k)
$$

in the strong operator topology in $\mathcal{L}\left(A_{\alpha}(\mathbb{D})\right)$, where $\left\{c_{k}\right\}_{k=-\infty}^{\infty}$ is a sequence of complex numbers. By [15, Lemma 5.1] the harmonic function

$$
h(z)=\sum_{k=-\infty}^{\infty} c_{k} r^{|k|} e^{i k \theta}, \quad z=r e^{i \theta} \in \mathbb{D},
$$

is bounded with uniform bound $|h(z)| \leq\|T\|$ for $z \in \mathbb{D}$. Passing to boundary values we obtain a function $f \in L^{\infty}(\mathbb{T})$ with Fourier coefficients $\hat{f}(k)=c_{k}$ for $k \in \mathbb{Z}$ (see for instance [20, Chapter 11]). Now

$$
T=\lim _{N \rightarrow \infty} \sum_{|k| \leq N}\left(1-\frac{|k|}{N+1}\right) \hat{f}(k) S_{\alpha}(k)=\int_{\mathbb{T}} f\left(e^{i \theta}\right) \mathrm{d} \omega_{S_{\alpha}}\left(e^{i \theta}\right)
$$

in the strong operator topology in $\mathcal{L}\left(A_{\alpha}(\mathbb{D})\right)$ by the continuity property $(2.9)$ for the functional calculus.

Conversely, if $T \in \mathcal{L}\left(A_{\alpha}(\mathbb{D})\right)$ has the form of an operator integral (2.10), then (2.12) holds and Proposition 2.1 gives that $T$ satisfies (0.4). The norm equality $\|T\|=\|f\|_{\infty}$ when $T$ has the form (2.10) holds more generally for an operator integral with respect to the shift operator on a Hilbert space of analytic functions on $\mathbb{D}$ (see $[15$, Theorem 5.2]). 
We wish to mention here also an interesting result by Conway and Ptak [10, Theorem 2.2] saying that if an operator $T \in \mathcal{L}(\mathcal{H})$ has an isometric $H^{\infty}(\mathbb{D})$ functional calculus and $\mathrm{d} \omega_{T}$ is absolutely continuous, then the $L^{\infty}(\mathbb{T})$ functional calculus $(2.8)$ is isometric. This generalizes the norm equality in Theorem 2.5.

For $k \geq 0$ we denote by $e_{k}$ the monomial

$$
e_{k}(z)=z^{k}, \quad z \in \mathbb{C} .
$$

Recall that the monomials $\left\{e_{k}\right\}_{k=0}^{\infty}$ form an orthogonal basis for $A_{\alpha}(\mathbb{D})$ such that $\left\|e_{k}\right\|_{\alpha}^{2}=\mu_{\alpha ; k}$ for $k \geq 0$.

Lemma 2.6. Let $\alpha>-1$ and $f \in L^{\infty}(\mathbb{T})$. Then

$$
\left\langle\int_{\mathbb{T}} f\left(e^{i \theta}\right) \mathrm{d} \omega_{S_{\alpha}}\left(e^{i \theta}\right) e_{k}, e_{j}\right\rangle_{\alpha}=\hat{f}(j-k) \mu_{\alpha ; \max (j, k)}
$$

for $j, k \geq 0$, where $\hat{f}(k)$ is the $k$-th Fourier coefficient of $f$.

Proof. A straightforward calculation shows that

$$
\left\langle S_{\alpha}(n) e_{k}, e_{j}\right\rangle_{\alpha}= \begin{cases}\mu_{\alpha ; \max (j, k)} & \text { for } n=j-k \\ 0 & \text { otherwise }\end{cases}
$$

By (2.9) we now have that

$$
\begin{aligned}
\left\langle\int_{\mathbb{T}} f\left(e^{i \theta}\right) \mathrm{d} \omega_{S_{\alpha}}\left(e^{i \theta}\right) e_{k}, e_{j}\right\rangle_{\alpha} & =\lim _{N \rightarrow \infty} \sum_{|n| \leq N}\left(1-\frac{|n|}{N+1}\right) \hat{f}(n)\left\langle S_{\alpha}(n) e_{k}, e_{j}\right\rangle_{\alpha} \\
& =\hat{f}(j-k) \mu_{\alpha ; \max (j, k)} .
\end{aligned}
$$

This completes the proof of the lemma.

\section{Toeplitz operators}

In this section we continue our study of operators $T \in \mathcal{L}\left(A_{\alpha}(\mathbb{D})\right)$ satisfying $(0.4)$ with particular emphasis on the relation to Toeplitz operators. By the Toeplitz operator on $A_{\alpha}(\mathbb{D})$ with bounded harmonic symbol $h$ we mean the operator $T_{h}$ defined by

$$
\left(T_{h} f\right)(z)=\int_{\mathbb{D}} \frac{1}{(1-\bar{\zeta} z)^{\alpha+2}} h(\zeta) f(\zeta) \mathrm{d} \mu_{\alpha}(\zeta), \quad z \in \mathbb{D},
$$

for $f \in A_{\alpha}(\mathbb{D})$. Notice that the integral kernel $1 /(1-\bar{\zeta} z)^{\alpha+2}$ in $(3.1)$ is the kernel function for $A_{\alpha}(\mathbb{D})$ and that formula (3.1) has the interpretation that the operator $T_{h}$ is the compression to $A_{\alpha}(\mathbb{D})$ of the operator of multiplication by $h$ on $L^{2}\left(\mathbb{D}, \mathrm{d} \mu_{\alpha}\right)$. It is well-known that $\left\|T_{h}\right\|=\sup _{z \in \mathbb{D}}|h(z)|$ for $h$ harmonic (see [25, Section 6.1]).

Trieu Le has pointed out to us that the statement of [15, Lemma 6.1] contains an unfortunate typo. The following lemma supplies the correct statement.

Lemma 3.1. Let $\alpha>-1$ and let $h$ be a bounded harmonic function in $\mathbb{D}$ with power series expansion

$$
h(z)=\sum_{k=-\infty}^{\infty} c_{k} r^{|k|} e^{i k \theta}, \quad z=r e^{i \theta} \in \mathbb{D} .
$$

Then

$$
\left\langle T_{h} e_{k}, e_{j}\right\rangle_{\alpha}=c_{j-k} \mu_{\alpha ; \max (j, k)}
$$

for $j, k \geq 0$, where $T_{h}$ is the Toeplitz operator on $A_{\alpha}(\mathbb{D})$ with symbol $h$. 
Proof. Recall the interpretation of $T_{h}$ as multiplication by $h$ followed by an orthogonal projection onto $A_{\alpha}(\mathbb{D})$. By straightforward calculation we have that

$$
\left\langle T_{h} e_{k}, e_{j}\right\rangle_{\alpha}=\int_{\mathbb{D}} h(z) z^{k} \bar{z}^{j} \mathrm{~d} \mu_{\alpha}(z)=c_{j-k} \mu_{\alpha ; \max (j, k)}
$$

for $j, k \geq 0$.

Recall the classical fact that bounded harmonic functions $h$ in $\mathbb{D}$ correspond to functions $f \in L^{\infty}(\mathbb{T})$ by means of the Poisson integral formula

$$
h(z)=P[f](z)=\frac{1}{2 \pi} \int_{\mathbb{T}} P\left(z e^{-i \theta}\right) f\left(e^{i \theta}\right) \mathrm{d} \theta, \quad z \in \mathbb{D},
$$

where $P(z)=\left(1-|z|^{2}\right) /|1-z|^{2}$ for $z \in \mathbb{D}$ is the Poisson kernel for $\mathbb{D}$. For $h$ given by (3.2) this correspondence means that $c_{k}=\hat{f}(k)$ for $k \in \mathbb{Z}$, where $\hat{f}(k)$ is the $k$-th Fourier coefficient of $f$. See for instance [20, Chapter 11] for details.

We next show that Toeplitz operators and functional calculus of shifts amount to the same class of objects.

Theorem 3.2. Let $\alpha>-1$ and $f \in L^{\infty}(\mathbb{T})$. Then

$$
T_{h}=\int_{\mathbb{T}} f\left(e^{i \theta}\right) \mathrm{d} \omega_{S_{\alpha}}\left(e^{i \theta}\right) \quad \text { in } \mathcal{L}\left(A_{\alpha}(\mathbb{D})\right),
$$

where $h=P[f]$ is the Poisson integral of $f$ and $T_{h}$ is the Toeplitz operator on $A_{\alpha}(\mathbb{D})$ with symbol $h$.

Proof. By Lemmas 2.6 and 3.1 we have that

$$
\left\langle T_{h} e_{k}, e_{j}\right\rangle_{\alpha}=\left\langle\int_{\mathbb{T}} f\left(e^{i \theta}\right) \mathrm{d} \omega_{S_{\alpha}}\left(e^{i \theta}\right) e_{k}, e_{j}\right\rangle_{\alpha}
$$

for $j, k \geq 0$. An approximation argument now yields the result.

We next characterize the Toeplitz operators using the operator identity (0.4).

Theorem 3.3. Let $\alpha>-1$ and let $T \in \mathcal{L}\left(A_{\alpha}(\mathbb{D})\right)$ be a bounded linear operator. Then $T$ satisfies $(0.4)$ if and only if $T$ is a Toeplitz operator $T=T_{h}$ on $A_{\alpha}(\mathbb{D})$ with bounded harmonic symbol $h$.

Proof. By Theorem 3.2 the Toeplitz operators on $A_{\alpha}(\mathbb{D})$ with bounded harmonic symbols and the operator integrals $\int_{\mathbb{T}} f \mathrm{~d} \omega_{S_{\alpha}}$ of functions $f \in L^{\infty}(\mathbb{T})$ amount to the same class of operators. The result now follows by Theorem 2.5 .

Given the information that an operator $T$ is a Toeplitz operator on $A_{\alpha}(\mathbb{D})$ with bounded harmonic symbol it is of interest to know how to calculate its symbol $h$ in (3.1) or equivalently the symbol $f$ in the operator integral representation (2.10). In Section 2 we described how to calculate these symbols from the homogeneous parts $T_{k}, k \in \mathbb{Z}$, of $T$. Another method is to calculate the Berezin transform $\tilde{T}$ of $T$ and use that $h(z)=\tilde{T}(z)$ for $z \in \mathbb{D}$ (see [25, Chapter 6] or [15, Proposition 5.2]).

We mention that for $n=\alpha+2$ positive integer the characterizations of operators $T \in \mathcal{L}\left(A_{\alpha}(\mathbb{D})\right)$ satisfying $(0.4)$ in terms of functional calculus for the shift (Theorem 2.5) or as Toeplitz operators (Theorem 3.3) originate from [15]. The novelty here is the extension of these results to the full parameter scale $\alpha>-1$. 


\section{Operator INEQUALities}

In this section we shall discuss two operator inequalities naturally derived from (0.2) and, as a consequence, we shall obtain (0.5). By the notation $T \in \mathcal{L}(\mathcal{H})$ we understand that $T$ is a bounded linear operator on a general not necessarily separable Hilbert space $\mathcal{H}$. For $\mathcal{H}$ a Hilbert space we denote by $\ell^{2}(\mathcal{H})$ the standard Hilbert space of square-summable sequences from $\mathcal{H}$ over an appropriate index set.

Let $\alpha>-1$. We shall consider left-invertible operators $T \in \mathcal{L}(\mathcal{H})$ such that

$$
\left(T^{*} T\right)^{-1} \leq \sum_{k \geq 0}(-1)^{k}\left(\begin{array}{l}
\alpha+2 \\
k+1
\end{array}\right) T^{k} T^{* k}
$$

with the sum convergent in the weak operator topology in $\mathcal{L}(\mathcal{H})$. The assumption of left-invertibility of $T$ is needed for the operator $\left(T^{*} T\right)^{-1}$ to exist in $\mathcal{L}(\mathcal{H})$. Inequality (4.1) is to be interpreted in the operator sense that

$$
\left\langle\left(T^{*} T\right)^{-1} x, x\right\rangle \leq \sum_{k \geq 0}(-1)^{k}\left(\begin{array}{c}
\alpha+2 \\
k+1
\end{array}\right)\left\|T^{* k} x\right\|^{2}, \quad x \in \mathcal{H} .
$$

The sum in (4.2) is always absolutely convergent since the coefficients $(-1)^{k}\left(\begin{array}{l}\alpha+2 \\ k+1\end{array}\right)$ are of constant sign for $k>\alpha+1$ as follows by Proposition 1.2.

Let $-1<\alpha \leq 0$. A related property of an operator $T \in \mathcal{L}(\mathcal{H})$ is that it satisfies the operator inequality

$$
\left\|x+\sum_{k \geq 1} C_{\alpha ; k} T^{k} x_{k}\right\|^{2} \leq(\alpha+2)\left(\|T x\|^{2}+\sum_{k \geq 1}\left\|x_{k}\right\|^{2}\right)
$$

for $x \in \mathcal{H}$ and $\left\{x_{k}\right\}_{k \geq 1} \in \ell^{2}(\mathcal{H})$ finitely supported, where $\left\{C_{\alpha ; k}\right\}_{k \geq 1}$ are arbitrary complex numbers such that $(0.6)$ holds. Notice that $(-1)^{k+1}\left(\begin{array}{l}\alpha+2 \\ k+1\end{array}\right) \geq 0$ for $k \geq 1$ by Proposition 1.2 which ensures existence of complex numbers $\left\{C_{\alpha ; k}\right\}_{k \geq 1}$ satisfying (0.6).

For the sake of easy reference we record that

$$
\left\|\left(T^{*} T\right)^{1 / 2} x\right\|^{2}=\left\langle\left(T^{*} T\right)^{1 / 2} x,\left(T^{*} T\right)^{1 / 2} x\right\rangle=\left\langle T^{*} T x, x\right\rangle=\|T x\|^{2}
$$

for $x \in \mathcal{H}$, where the positive square root is used.

We shall next investigate the relation between (4.1) and (4.3).

Theorem 4.1. Let $-1<\alpha \leq 0$ and let $T \in \mathcal{L}(\mathcal{H})$ be a left-invertible operator satisfying (4.1). Then $T$ satisfies (4.3).

Proof. Notice first that (4.1) is equivalent to

$$
\left\|\left(T^{*} T\right)^{-1 / 2} x\right\|^{2}+\sum_{k \geq 1}\left\|\bar{C}_{\alpha ; k} T^{* k} x\right\|^{2} \leq(\alpha+2)\|x\|^{2}, \quad x \in \mathcal{H} .
$$

Now introduce the operator $M$ on $\mathcal{H}$ by $M x=\left\{y_{k}\right\}_{k \geq 0}$, where $y_{0}=\left(T^{*} T\right)^{-1 / 2} x$ and $y_{k}=\bar{C}_{\alpha ; k} T^{* k} x$ for $k \geq 1$. By (4.5) the operator $M$ maps $\mathcal{H}$ boundedly into $\ell^{2}(\mathcal{H})$ and $\|M\|^{2} \leq \alpha+2$. Passing to the adjoint we have that $M^{*}$ maps $\ell^{2}(\mathcal{H})$ into $\mathcal{H}$ and $\left\|M^{*}\right\|^{2} \leq \alpha+2$. A calculation gives that

$$
M^{*}\left(\left\{x_{k}\right\}_{k \geq 0}\right)=\left(T^{*} T\right)^{-1 / 2} x_{0}+\sum_{k \geq 1} C_{\alpha ; k} T^{k} x_{k}
$$


for $\left\{x_{k}\right\}_{k \geq 0} \in \ell^{2}(\mathcal{H})$ finitely supported. This gives the inequality that

$$
\left\|\left(T^{*} T\right)^{-1 / 2} x_{0}+\sum_{k \geq 1} C_{\alpha ; k} T^{k} x_{k}\right\|^{2} \leq(\alpha+2) \sum_{k \geq 0}\left\|x_{k}\right\|^{2}
$$

for $\left\{x_{k}\right\}_{k \geq 0} \in \ell^{2}(\mathcal{H})$ finitely supported. Setting $x=\left(T^{*} T\right)^{-1 / 2} x_{0}$ in (4.6) using (4.4) we obtain the conclusion of the theorem.

As a consequence we obtain (0.5) for the Bergman shift operator.

Corollary 4.2. Let $-1<\alpha \leq 0$ and let $\mathcal{E}$ be a Hilbert space. Then the shift operator $S_{\alpha}$ on $A_{\alpha}(\mathcal{E})$ satisfies the inequality

$$
\left\|f+\sum_{k \geq 1} C_{\alpha ; k} S_{\alpha}^{k} f_{k}\right\|_{\alpha}^{2} \leq(\alpha+2)\left(\left\|S_{\alpha} f\right\|_{\alpha}^{2}+\sum_{k \geq 1}\left\|f_{k}\right\|_{\alpha}^{2}\right)
$$

for all $f \in A_{\alpha}(\mathcal{E})$ and $\left\{f_{k}\right\}_{k \geq 1} \in \ell^{2}\left(A_{\alpha}(\mathcal{E})\right)$ finitely supported, where the $C_{\alpha ; k}$ 's are as in (0.6).

Proof. Recall Theorem 1.4. An application of Theorem 4.1 now yields the conclusion of the corollary.

We next turn to the converse of Theorem 4.1.

Theorem 4.3. Let $-1<\alpha \leq 0$. Let $T \in \mathcal{L}(\mathcal{H})$ be an operator satisfying (4.3). Then $T$ is left-invertible and satisfies (4.1).

Proof. Setting $x_{k}=0$ for $k \geq 1$ in (4.3) we see that $\|x\|^{2} \leq(\alpha+2)\|T x\|^{2}$ for $x \in \mathcal{H}$, showing that $T$ is left-invertible.

Using the substitution $x=\left(T^{*} T\right)^{-1 / 2} x_{0}$ and (4.4) in (4.3) we get

$$
\left\|\left(T^{*} T\right)^{-1 / 2} x_{0}+\sum_{k \geq 1} C_{\alpha ; k} T^{k} x_{k}\right\|^{2} \leq(\alpha+2) \sum_{k \geq 0}\left\|x_{k}\right\|^{2}
$$

for $\left\{x_{k}\right\}_{k \geq 0} \in \ell^{2}(\mathcal{H})$ finitely supported. Introduce now the operator $N$ by

$$
N\left(\left\{x_{k}\right\}_{k \geq 0}\right)=\left(T^{*} T\right)^{-1 / 2} x_{0}+\sum_{k \geq 1} C_{\alpha ; k} T^{k} x_{k}
$$

for $\left\{x_{k}\right\}_{k \geq 0} \in \ell^{2}(\mathcal{H})$ finitely supported. It is clear that $N$ extends uniquely by continuity to a bounded linear operator from $\ell^{2}(\mathcal{H})$ into $\mathcal{H}$ with $\|N\|^{2} \leq \alpha+2$. Passing to the adjoint we have that $N^{*}$ is a bounded operator from $\mathcal{H}$ into $\ell^{2}(\mathcal{H})$ with norm control $\left\|N^{*}\right\|^{2} \leq \alpha+2$. A calculation shows that $N^{*} x=\left\{y_{k}\right\}_{k \geq 0}$, where $y_{0}=\left(T^{*} T\right)^{-1 / 2} x$ and $y_{k}=\bar{C}_{\alpha ; k} T^{* k} x$ for $k \geq 1$. This gives

$$
\left\|\left(T^{*} T\right)^{-1 / 2} x\right\|^{2}+\sum_{k \geq 1}\left|C_{\alpha ; k}\right|^{2}\left\|T^{* k} x\right\|^{2} \leq(\alpha+2)\|x\|^{2}, \quad x \in \mathcal{H} .
$$

By this last inequality (4.1) follows.

We wish to mention that Theorems 4.1 and 4.3 are extracted from the method of proof of Shimorin [21, Theorem 3.6]. A similar analysis can be found in McCullough and Richter [16, Section 3].

For a left-invertible operator $T \in \mathcal{L}(\mathcal{H})$ we denote by $T^{\prime}=T\left(T^{*} T\right)^{-1}$ its Cauchy dual. We shall need also the following estimate of powers of $T^{\prime}$. 
Proposition 4.4. Let $-1<\alpha \leq 0$ and let $T \in \mathcal{L}(\mathcal{H})$ be a left-invertible operator satisfying (4.1). Then

$$
\begin{aligned}
\left\|T^{k} x\right\|^{2} \leq & \left(\begin{array}{c}
k+\alpha \\
k-1
\end{array}\right)\left(\left\|T^{\prime} x\right\|^{2}-\|x\|^{2}\right) \\
& +\left(\left(\begin{array}{c}
k+\alpha+1 \\
k
\end{array}\right)-(\alpha+1)\left(\begin{array}{c}
k+\alpha \\
k-1
\end{array}\right)\right)\|x\|^{2}, \quad x \in \mathcal{H},
\end{aligned}
$$

for $k=2,3, \ldots$, where $T^{\prime}$ is the Cauchy dual of $T$.

Proof. Let $x \in \mathcal{H}$. We shall consider the sequence

$$
a_{m}=\left\|T^{\prime m} x\right\|^{2}, \quad m=0,1,2, \ldots,
$$

of non-negative real numbers. Recall that $T^{*} T^{\prime}=\left(T^{*} T\right)^{-1}$. Substituting $T^{\prime m} x$ for $x$ in (4.2) we have

$$
\begin{aligned}
\left\|T^{\prime m+1} x\right\|^{2} & \leq \sum_{k=0}^{m}(-1)^{k}\left(\begin{array}{l}
\alpha+2 \\
k+1
\end{array}\right)\left\|T^{\prime m-k} x\right\|^{2}+\sum_{k=m+1}^{\infty}(-1)^{k}\left(\begin{array}{c}
\alpha+2 \\
k+1
\end{array}\right)\left\|T^{*(k-m)} x\right\|^{2} \\
& \leq \sum_{k=0}^{m}(-1)^{k}\left(\begin{array}{l}
\alpha+2 \\
k+1
\end{array}\right)\left\|T^{\prime m-k} x\right\|^{2}
\end{aligned}
$$

since $T^{*} T^{\prime}=I$, where the last inequality follows by an estimation using Proposition 1.2. For the numbers $\left\{a_{m}\right\}_{m \geq 0}$ this gives the inequalities

$$
a_{m+1} \leq \sum_{k=0}^{m}(-1)^{k}\left(\begin{array}{l}
\alpha+2 \\
k+1
\end{array}\right) a_{m-k}, \quad m=0,1,2, \ldots
$$

We now introduce numbers $b_{m}$ such that

$$
a_{m+1}+b_{m}=\sum_{k=0}^{m}(-1)^{k}\left(\begin{array}{l}
\alpha+2 \\
k+1
\end{array}\right) a_{m-k}, \quad m=0,1,2, \ldots
$$

Notice that $b_{m} \geq 0$ for $m \geq 0$. Passing to generating functions we have

$$
\frac{A(z)-A(0)}{z}+B(z)=\left(\sum_{k=0}^{\infty}(-1)^{k}\left(\begin{array}{l}
\alpha+2 \\
k+1
\end{array}\right) z^{k}\right) A(z)
$$

for $|z|$ small, where

$$
A(z)=\sum_{k=0}^{\infty} a_{k} z^{k} \quad \text { and } \quad B(z)=\sum_{k=0}^{\infty} b_{k} z^{k} .
$$

Solving for $A(z)$ in (4.7) using the binomial series (1.3) we have that

$$
A(z)=\frac{A(0)-z B(z)}{(1-z)^{\alpha+2}} .
$$

Now

$$
A(z)=\frac{a_{0}-b_{0} z}{(1-z)^{\alpha+2}}-\frac{z\left(B(z)-b_{0}\right)}{(1-z)^{\alpha+2}}
$$

and the rightmost function in this last equality has non-negative Taylor coefficients since $b_{m} \geq 0$ for $m \geq 1$. This leads to the estimate

$$
a_{m} \leq\left(\begin{array}{c}
m+\alpha+1 \\
m
\end{array}\right) a_{0}-\left(\begin{array}{c}
m+\alpha \\
m-1
\end{array}\right) b_{0}, \quad m=2,3, \ldots
$$


Using $a_{1}+b_{0}=(\alpha+2) a_{0}$ we calculate that

$$
\begin{aligned}
a_{m} & \leq\left(\begin{array}{c}
m+\alpha+1 \\
m
\end{array}\right) a_{0}-\left(\begin{array}{c}
m+\alpha \\
m-1
\end{array}\right)\left((\alpha+2) a_{0}-a_{1}\right) \\
& =\left(\begin{array}{c}
m+\alpha \\
m-1
\end{array}\right)\left(a_{1}-a_{0}\right)+\left(\left(\begin{array}{c}
m+\alpha+1 \\
m
\end{array}\right)-(\alpha+1)\left(\begin{array}{c}
m+\alpha \\
m-1
\end{array}\right)\right) a_{0}
\end{aligned}
$$

for $m=2,3, \ldots$ This yields the conclusion of the proposition.

For $\alpha=0$ Proposition 4.4 gives the bounds

$$
\left\|T^{k} x\right\|^{2} \leq k\left(\left\|T^{\prime} x\right\|^{2}-\|x\|^{2}\right)+\|x\|^{2}, \quad x \in \mathcal{H},
$$

for $k=2,3, \ldots$, which were used in [17] to prove Cesàro summability in a context of wandering subspace theorems.

\section{KeRnel FUnCtions}

Let $\mathcal{E}$ be a Hilbert space. By a Hilbert space of $\mathcal{E}$-valued analytic functions in a domain $\Omega$ we mean a Hilbert space $\mathfrak{H}$ whose elements $f$ are $\mathcal{E}$-valued analytic functions in $\Omega$ in such a way that the point evaluations

$$
\mathfrak{H} \ni f \mapsto f(\zeta) \in \mathcal{E}
$$

at points $\zeta \in \Omega$ are all continuous. Associated to such a space $\mathfrak{H}$ there is a reproducing kernel function $K_{\mathfrak{H}}: \Omega \times \Omega \rightarrow \mathcal{L}(\mathcal{E})$ uniquely determined by the properties that $K_{\mathfrak{H}}(\cdot, \zeta) e \in \mathfrak{H}$ and

$$
\langle f(\zeta), e\rangle=\left\langle f, K_{\mathfrak{H}}(\cdot, \zeta) e\right\rangle, \quad f \in \mathfrak{H},
$$

for all $\zeta \in \Omega$ and $e \in \mathcal{E}$. The essential property of a kernel function is that of positive definiteness and it is well-known that the kernel function determines the space completely (see [5]). As an example of kernel functions we mention the Bergman kernels $K_{\alpha}$ from Section 1.

Assuming that the shift operator

$$
S f(z)=z f(z), \quad z \in \Omega
$$

operates on a Hilbert space $\mathfrak{H}$ of $\mathcal{E}$-valued analytic functions on $\Omega$ it is well-known that the adjoint shift $S^{*}$ acts on reproducing elements by

$$
S^{*} K_{\mathfrak{H}}(\cdot, \zeta) e=\bar{\zeta} K_{\mathfrak{H}}(\cdot, \zeta) e
$$

for $\zeta \in \Omega$ and $e \in \mathcal{E}$. We next consider the backward shift.

Lemma 5.1. Let $\mathfrak{H}$ be a Hilbert space of $\mathcal{E}$-valued analytic functions on a domain $\Omega$ containing the origin such that the backward shift operator

$$
B f(z)=(f(z)-f(0)) / z, \quad z \in \Omega,
$$

operates in $\mathfrak{H}$. Then

$$
B^{*} K_{\mathfrak{H}}(\cdot, \zeta) e=\left(K_{\mathfrak{H}}(\cdot, \zeta) e-K_{\mathfrak{H}}(\cdot, 0) e\right) / \bar{\zeta}
$$

for $\zeta \in \Omega$ and $e \in \mathcal{E}$. 
Proof. Let $e_{1}, e_{2} \in \mathcal{E}$ and set $f=B^{*} K_{\mathfrak{H}}(\cdot, \zeta) e_{1}$. Using the reproducing property (5.1) we calculate that

$$
\begin{aligned}
\left\langle f(z), e_{2}\right\rangle & =\left\langle B^{*} K_{\mathfrak{H}}(\cdot, \zeta) e_{1}, K_{\mathfrak{H}}(\cdot, z) e_{2}\right\rangle=\left\langle K_{\mathfrak{H}}(\cdot, \zeta) e_{1}, B K_{\mathfrak{H}}(\cdot, z) e_{2}\right\rangle \\
& =\overline{\left\langle B K_{\mathfrak{H}}(\cdot, z) e_{2}, K_{\mathfrak{H}}(\cdot, \zeta) e_{1}\right\rangle}=\overline{\left\langle\left(K_{\mathfrak{H}}(\zeta, z) e_{2}-K_{\mathfrak{H}}(0, z) e_{2}\right) / \zeta, e_{1}\right\rangle} \\
& =\left\langle\left(K_{\mathfrak{H}}(z, \zeta) e_{1}-K_{\mathfrak{H}}(z, 0) e_{1}\right) / \bar{\zeta}, e_{2}\right\rangle
\end{aligned}
$$

for $z, \zeta \in \Omega$. This yields the conclusion of the lemma.

Let us recall briefly the so-called Wold map $V$ from $[4,21]$ which is a convenient device for the purpose of modeling a general Hilbert space operator as a shift operator on a Hilbert space of analytic functions. Let $T \in \mathcal{L}(\mathcal{H})$ be a left-invertible operator and denote by $L=\left(T^{*} T\right)^{-1} T^{*}$ its Moore-Penrose left-inverse. The operator $P=I-T L$ is the orthogonal projection of $\mathcal{H}$ onto the wandering subspace $\mathcal{E}=\mathcal{H} \ominus T(\mathcal{H})=\operatorname{ker} T^{*}$ for $T$. For $x \in \mathcal{H}$ we consider the $\mathcal{E}$-valued analytic function

$$
V x(z)=P(I-z L)^{-1} x=\sum_{k \geq 0}\left(P L^{k} x\right) z^{k}, \quad|z|<1 / \rho(L),
$$

where $\rho(L)$ is the spectral radius of $L$. The map $V: x \mapsto V x$ has the intertwining properties

$$
V T x(z)=z V x(z) \quad \text { and } \quad V L x(z)=(V x(z)-V x(0)) / z
$$

for $|z|<1 / \rho(L)$. In other words, the map $V$ intertwines $T$ with the shift $S$ and $L$ with the backward shift $B$. The map $V: x \mapsto V x$ being injective corresponds to the operator $T$ being pure in the sense that

$$
\bigcap_{k \geq 0} T^{k}(\mathcal{H})=\{0\}
$$

(see [21, Lemma 2.2]). For $T$ pure we equip the range $\mathfrak{H}=V(\mathcal{H})$ with the norm induced by the map $V$, that is, we set $\|f\|^{2}=\|x\|^{2}$ when $f=V x$ for some $x \in \mathcal{H}$. This norm makes $\mathfrak{H}$ a Hilbert space of $\mathcal{E}$-valued analytic functions and the map $V: \mathcal{H} \rightarrow \mathfrak{H}$ unitary.

The following proposition is from Shimorin [21, Proposition 2.13]. For the sake of completeness we include a proof.

Proposition 5.2. Let $T \in \mathcal{L}(\mathcal{H})$ be a pure left-invertible operator and $\mathfrak{H}=V(\mathcal{H})$ as above. Then the kernel function for $\mathfrak{H}$ is given by the formula

$$
K_{\mathfrak{H}}(z, \zeta)=\left.P(I-z L)^{-1}\left(I-\bar{\zeta} L^{*}\right)^{-1}\right|_{\mathcal{E}}
$$

for $|z|,|\zeta|<1 / \rho(L)$. In particular $K_{\mathfrak{H}}(\cdot, 0)=I_{\mathcal{E}}$, where $I_{\mathcal{E}}$ is the identity operator on $\mathcal{E}$.

Proof. For $f=V x \in \mathfrak{H}, x \in \mathcal{H}, e \in \mathcal{E}$ and $|\zeta|<1 / \rho(L)$ we have

$$
\begin{aligned}
\langle f(\zeta), e\rangle & =\left\langle P(I-\zeta L)^{-1} x, e\right\rangle=\left\langle x,\left(I-\bar{\zeta} L^{*}\right)^{-1} e\right\rangle=\left\langle V x, V\left(I-\bar{\zeta} L^{*}\right)^{-1} e\right\rangle \\
& =\left\langle f, P(I-\cdot L)^{-1}\left(I-\bar{\zeta} L^{*}\right)^{-1} e\right\rangle .
\end{aligned}
$$

This gives the formula for $K_{\mathfrak{H}}$. The property $K_{\mathfrak{H}}(\cdot, 0)=I_{\mathcal{E}}$ is evident by the formula.

Let us comment on the property $K_{\mathfrak{H}}(\cdot, 0)=I_{\mathcal{E}}$ of a kernel function. 
Proposition 5.3. Let $\mathfrak{H}$ be a Hilbert space of $\mathcal{E}$-valued analytic functions in a domain $\Omega$ containing the origin. Then

$$
K_{\mathfrak{H}}(z, 0)=I_{\mathcal{E}}, \quad z \in \Omega,
$$

if and only if the space $\mathcal{E}$ identified as constant functions on $\Omega$ is isometrically contained in $\mathfrak{H}$ in such a way that $\mathfrak{H}$ admits the orthogonal sum decomposition

$$
\mathfrak{H}=\mathcal{E} \oplus\{f \in \mathfrak{H}: \quad f(0)=0\} .
$$

Proof. Assume first that $K_{\mathfrak{H}}(\cdot, 0)=I_{\mathcal{E}}$. It is straightforward to check that the constant functions are isometrically contained in $\mathfrak{H}$. A straightforward calculation using the reproducing property (5.1) gives

$$
\mathfrak{H} \ominus \mathcal{E}=\{f \in \mathfrak{H}: \quad f(0)=0\},
$$

which yields the orthogonal sum decomposition.

Assume next that $\mathcal{E}$ is isometrically contained in $\mathfrak{H}$ in such a way that (5.3) holds. We show first that $K_{\mathfrak{H}}(0,0) \geq I_{\mathcal{E}}$ in $\mathcal{L}(\mathcal{E})$. For this purpose let $f \equiv e$ be a constant function in $\mathfrak{H}$. By the reproducing property $(5.1)$ we have

$$
\|e\|^{2}=\langle f(0), e\rangle=\left\langle f, K_{\mathfrak{H}}(\cdot, 0) e\right\rangle \leq\|f\|\left\|K_{\mathfrak{H}}(\cdot, 0) e\right\|=\|f\|\left\langle K_{\mathfrak{H}}(0,0) e, e\right\rangle^{1 / 2}
$$

using Cauchy-Schwarz inequality. Since $\|f\|=\|e\|$, a cancellation argument gives $\|e\|^{2} \leq\left\langle K_{\mathfrak{H}}(0,0) e, e\right\rangle$ for $e \in \mathcal{E}$, which proves the assertion that $K_{\mathfrak{H}}(0,0) \geq I_{\mathcal{E}}$.

We now show that $K_{\mathfrak{H}}(\cdot, 0)=I_{\mathcal{E}}$. Consider a function $f \in \mathfrak{H}$ of the form $f=K_{\mathfrak{H}}(\cdot, 0) e$ for some $e \in \mathcal{E}$. By (5.3) we have $\|f\|^{2}=\|f-f(0)\|^{2}+\|f(0)\|^{2}$. Using the reproducing property (5.1) we have

$$
\begin{aligned}
\|f-f(0)\|^{2} & =\|f\|^{2}-\|f(0)\|^{2}=\left\langle K_{\mathfrak{H}}(0,0) e, e\right\rangle-\left\|K_{\mathfrak{H}}(0,0) e\right\|^{2} \\
& =\left\langle K_{\mathfrak{H}}(0,0)\left(I_{\mathcal{E}}-K_{\mathfrak{H}}(0,0)\right) e, e\right\rangle \leq 0,
\end{aligned}
$$

where the last inequality follows from the inequality $K_{\mathfrak{H}}(0,0) \geq I_{\mathcal{E}}$ shown in the previous paragraph. As a consequence $f=f(0)$ is constant. Varying $e \in \mathcal{E}$ we see that $K_{\mathfrak{H}}(\cdot, 0)=K_{\mathfrak{H}}(0,0)$. Another look at $(5.4)$ reveals that $K_{\mathfrak{H}}(0,0)^{2}=K_{\mathfrak{H}}(0,0)$ by a polarization argument, showing that the operator $K_{\mathfrak{H}}(0,0)$ is an orthogonal projection. Since $K_{\mathfrak{H}}(0,0) \geq I_{\mathcal{E}}$, this gives that $K_{\mathfrak{H}}(0,0)=I_{\mathcal{E}}$. We now conclude that $K_{\mathfrak{H}}(\cdot, 0)=I_{\mathcal{E}}$.

Let $\alpha>-1$. We shall next consider left-invertible operators $T \in \mathcal{L}(\mathcal{H})$ such that

$$
\left(T^{*} T\right)^{-1}=\sum_{k \geq 0}(-1)^{k}\left(\begin{array}{l}
\alpha+2 \\
k+1
\end{array}\right) T^{k} T^{* k}
$$

with convergence in the weak operator topology in $\mathcal{L}(\mathcal{H})$. By a polarization argument (5.5) is equivalent to

$$
\left\langle\left(T^{*} T\right)^{-1} x, x\right\rangle=\sum_{k \geq 0}(-1)^{k}\left(\begin{array}{c}
\alpha+2 \\
k+1
\end{array}\right)\left\|T^{* k} x\right\|^{2}, \quad x \in \mathcal{H},
$$

and this last sum is absolutely convergent since the coefficients $(-1)^{k}\left(\begin{array}{l}\alpha+2 \\ k+1\end{array}\right)$ are of constant sign for $k>\alpha+1$ by Proposition 1.2.

Theorem 5.4. Let $\alpha>-1$. Let $T \in \mathcal{L}(\mathcal{H})$ be a pure left-invertible operator satisfying $(5.5)$, and set $\mathfrak{H}=V(\mathcal{H})$. Then $\mathfrak{H}=A_{\alpha}(\mathcal{E})$ is the standard weighted Bergman space with weight parameter $\alpha$ and multiplicity $\mathcal{E}=\mathcal{H} \ominus T(\mathcal{H})$. In particular, the operator $T$ is unitarily equivalent to a vector-valued Bergman shift $S_{\alpha}$. 
Proof. We calculate the kernel function $K_{\mathfrak{H}}$ from Proposition 5.2. Observe first that

$$
K_{\mathfrak{H}}(z, \zeta)=I_{\mathcal{E}}+\bar{\zeta} z\left(\left.\sum_{j, k \geq 0} P L^{j+1} L^{*(k+1)}\right|_{\mathcal{E}} \bar{\zeta}^{k} z^{j}\right)
$$

for $|\zeta|,|z|<1 / \rho(L)$. Recall that $L L^{*}=\left(T^{*} T\right)^{-1}$. Using (5.5) we calculate

$$
\begin{aligned}
\left.P L^{j+1} L^{*(k+1)}\right|_{\mathcal{E}} & =\left.\sum_{n \geq 0}(-1)^{n}\left(\begin{array}{l}
\alpha+2 \\
n+1
\end{array}\right) P L^{j} T^{n} T^{* n} L^{* k}\right|_{\mathcal{E}} \\
& =\left.\sum_{n=0}^{\min (j, k)}(-1)^{n}\left(\begin{array}{c}
\alpha+2 \\
n+1
\end{array}\right) P L^{j-n} L^{*(k-n)}\right|_{\mathcal{E}}
\end{aligned}
$$

using that $L T=I$ and $P T=0$. Going back to (5.6) we have

$$
\begin{aligned}
K_{\mathfrak{H}}(z, \zeta) & =I_{\mathcal{E}}+\bar{\zeta} z\left(\left.\sum_{j, k \geq 0} \sum_{n=0}^{\min (j, k)}(-1)^{n}\left(\begin{array}{l}
\alpha+2 \\
n+1
\end{array}\right) P L^{j-n} L^{*(k-n)}\right|_{\mathcal{E}} \bar{\zeta}^{k} z^{j}\right) \\
& =I_{\mathcal{E}}+\bar{\zeta} z\left(\left.\sum_{n \geq 0} \sum_{j, k \geq n}(-1)^{n}\left(\begin{array}{l}
\alpha+2 \\
n+1
\end{array}\right) P L^{j-n} L^{*(k-n)}\right|_{\mathcal{E}} \bar{\zeta}^{k} z^{j}\right) \\
& =I_{\mathcal{E}}+\bar{\zeta} z\left(\sum_{n \geq 0}(-1)^{n}\left(\begin{array}{l}
\alpha+2 \\
n+1
\end{array}\right) \bar{\zeta}^{n} z^{n}\right) K_{\mathfrak{H}}(z, \zeta)
\end{aligned}
$$

for $|\zeta|,|z|<1 / \rho(L)$ by changes of order of summation. Using the binomial series (1.3) we see that

$$
K_{\mathfrak{H}}(z, \zeta)=I_{\mathcal{E}}-\left((1-\bar{\zeta} z)^{\alpha+2}-1\right) K_{\mathfrak{H}}(z, \zeta)
$$

for $|\zeta|,|z|<1 / \rho(L)$. Solving for $K_{\mathfrak{H}}$ we conclude that $K_{\mathfrak{H}}(z, \zeta)=1 /(1-\bar{\zeta} z)^{\alpha+2} I_{\mathcal{E}}$ for $|\zeta|,|z|<1 / \rho(L)$, that is, $K_{\mathfrak{H}}$ is the kernel function for $A_{\alpha}(\mathcal{E})$. By the well-known fact that a Hilbert space of analytic functions is completely determined by its kernel function we arrive at the conclusion that $\mathfrak{H}=A_{\alpha}(\mathcal{E})$ (see [5, Section I.2]).

We mention that Theorem 5.4 generalizes a recent result from Giselsson and Olofsson [12, Theorem 3.1] for $n=\alpha+2$ positive integer to the full parameter scale $\alpha>-1$. In conclusion, the operator identity (5.5) together with a pureness condition characterize the Bergman shift operator $S_{\alpha}$ up to unitary equivalence allowing for a general multiplicity.

We next consider operators satisfying the operator inequality (4.1).

Theorem 5.5. Let $\alpha>-1$. Let $T \in \mathcal{L}(\mathcal{H})$ be a pure left-invertible operator and set $\mathfrak{H}=V(\mathcal{H})$. Assume also that $\rho(L) \leq 1$. Then $T$ satisfies (4.1) if and only if the kernel function for $\mathfrak{H}$ admits the representation

$$
K_{\mathfrak{H}}(z, \zeta)=\frac{1}{(1-\bar{\zeta} z)^{2+\alpha}}\left(I_{\mathcal{E}}-\bar{\zeta} z \ell(z, \zeta)\right), \quad(z, \zeta) \in \mathbb{D}^{2},
$$

for some positive definite sesqui-analytic $\mathcal{L}(\mathcal{E})$-valued kernel $\ell$ in $\mathbb{D}^{2}$.

Proof. The assumption $\rho(L) \leq 1$ ensures that $\mathfrak{H}$ is a Hilbert space of analytic functions in $\mathbb{D}$. Recall that $L L^{*}=\left(T^{*} T\right)^{-1}$. Observe also that $V T^{*}=S^{*} V$ which follows from the intertwining relation $V T=S V$ since the map $V: \mathcal{H} \rightarrow \mathfrak{H}$ is 
unitary. Similarly $V L^{*}=B^{*} V$, where $B$ is the backward shift on $\mathfrak{H}$. Applying the map $V: \mathcal{H} \rightarrow \mathfrak{H}$ we see that (4.1) equivalently says that

$$
\left\|B^{*} f\right\|^{2} \leq \sum_{n \geq 0}(-1)^{n}\left(\begin{array}{l}
\alpha+2 \\
n+1
\end{array}\right)\left\|S^{* n} f\right\|^{2}, \quad f \in \mathfrak{H} .
$$

Recall also that the sign of coefficients in (5.8) is constant for $n>\alpha+1$ by Proposition 1.2 showing that the sum is absolutely convergent.

We shall next evaluate $(5.8)$ on an element $f$ in $\mathfrak{H}$ of the form

$$
f=\sum_{j=1}^{N} K_{\mathfrak{H}}\left(\cdot, \zeta_{j}\right) e_{j}
$$

where $\zeta_{1}, \ldots, \zeta_{N} \in \mathbb{D}$ and $e_{1}, \ldots, e_{N} \in \mathcal{E}$. Observe that

$$
B^{*} f=\sum_{j=1}^{N}\left(K_{\mathfrak{H}}\left(\cdot, \zeta_{j}\right) e_{j}-I_{\mathcal{E}} e_{j}\right) / \bar{\zeta}_{j}
$$

by Lemma 5.1 and Proposition 5.2. Observe also that

$$
S^{* n} f=\sum_{j=1}^{N} \bar{\zeta}_{j}^{n} K_{\mathfrak{H}}\left(\cdot, \zeta_{j}\right) e_{j}
$$

for $n \geq 0$ by (5.2). Substituting (5.9) into (5.8) we get

$$
\begin{aligned}
\sum_{j, k=1}^{N} \frac{1}{\bar{\zeta}_{j} \zeta_{k}} & \left(\left\langle K_{\mathfrak{H}}\left(\zeta_{k}, \zeta_{j}\right) e_{j}, e_{k}\right\rangle-\left\langle I_{\mathcal{E}} e_{j}, e_{k}\right\rangle\right) \\
& \leq \sum_{n \geq 0}(-1)^{n}\left(\begin{array}{l}
\alpha+2 \\
n+1
\end{array}\right) \sum_{j, k=1}^{N} \bar{\zeta}_{j}^{n} \zeta_{k}^{n}\left\langle K_{\mathfrak{H}}\left(\zeta_{k}, \zeta_{j}\right) e_{j}, e_{k}\right\rangle
\end{aligned}
$$

which simplifies to

$$
\begin{aligned}
& \sum_{j, k=1}^{N} \frac{1}{\bar{\zeta}_{j} \zeta_{k}}\left(\left\langle K_{\mathfrak{H}}\left(\zeta_{k}, \zeta_{j}\right) e_{j}, e_{k}\right\rangle-\left\langle I_{\mathcal{E}} e_{j}, e_{k}\right\rangle\right) \\
& \leq \sum_{j, k=1}^{N} \frac{1-\left(1-\bar{\zeta}_{j} \zeta_{k}\right)^{\alpha+2}}{\bar{\zeta}_{j} \zeta_{k}}\left\langle K_{\mathfrak{H}}\left(\zeta_{k}, \zeta_{j}\right) e_{j}, e_{k}\right\rangle
\end{aligned}
$$

after a change of order of summation and use of the binomial series (1.3).

Introduce now the $\mathcal{L}(\mathcal{E})$-valued function

$$
\ell(z, \zeta)=\frac{1-(1-\bar{\zeta} z)^{\alpha+2}}{\bar{\zeta} z} K_{\mathfrak{H}}(z, \zeta)-\frac{1}{\bar{\zeta} z}\left(K_{\mathfrak{H}}(z, \zeta)-I_{\mathcal{E}}\right), \quad(z, \zeta) \in \mathbb{D}^{2}
$$

Observe that the function $\ell$ is sesqui-analytic by Proposition 5.2. Notice that (5.10) can be restated as

$$
\sum_{j, k=1}^{N}\left\langle\ell\left(\zeta_{k}, \zeta_{j}\right) e_{j}, e_{k}\right\rangle \geq 0
$$

Varying $f \in \mathfrak{H}$ over the dense set of elements of the form (5.9) we conclude that (5.8) is equivalent to positive definiteness of $\ell$. Solving for $K_{\mathfrak{H}}$ in terms of $\ell$ the representation formula (5.7) follows. 
Remark 5.6. The assumption $\rho(L) \leq 1$ in Theorem 5.5 is inserted to ensure that $\mathfrak{H}$ is a Hilbert space of analytic functions in $\mathbb{D}$. In the restricted parameter range $-1<\alpha \leq 0$, the spectral radius control $\rho(L) \leq 1$ is a consequence of (4.1) as follows by Proposition 4.4 and the spectral radius formula. Elaborating the proof of Proposition 4.4 we can more generally show that $\rho(L) \leq 1$ follows from (4.1) when $2 m-1 \leq \alpha \leq 2 m$ for some integer $m \geq 0$. The details are omitted.

For $\alpha=0$ the structure formula (5.7) goes back to Shimorin [21, formula (3.4)]; see also [13, Section 6].

For a general result along similar lines as Theorem 5.5, see McCullough and Richter [16, Theorem 3.2]. From the point of view of normalized reproducing kernels for general shift invariant subspaces of $A_{\alpha}(\mathbb{D})$ structure formulas of the form $(5.7)$ with $\ell$ positive definite seem relevant only in the parameter range $-1<\alpha \leq 0$, see McCullough and Richter [16].

\section{REFERENCES}

[1] Ahern P, On the range of the Berezin transform, J. Funct. Anal. 215 (2004) 206-216.

[2] Ahern P, Čučković Ž, A theorem of Brown-Halmos type for Bergman space Toeplitz operators, J. Funct. Anal. 187 (2001) 200-210.

[3] Aleman A, Richter S, Single point extremal functions in Bergman-type spaces, Indiana Univ. Math. J. 51 (2002) 581-605.

[4] Aleman A, Richter S, Sundberg C, Beurling's theorem for the Bergman space, Acta Math. 177 (1996) 275-310.

[5] Aronszajn N, Theory of reproducing kernels, Trans. Amer. Math. Soc. 68 (1950) 337-404.

[6] Artin E, The gamma function, Holt, Rinehart and Winston, 1964.

[7] Axler S, Čučković Ž, Commuting Toeplitz operators with harmonic symbols, Integral Equations Operator Theory 14 (1991) 1-12.

[8] Brown A, Halmos PR, Algebraic properties of Toeplitz operators, J. Reine Angew. Math. 213 (1963/1964) 89-102.

[9] Chailos G, Reproducing kernels and invariant subspaces of the Bergman shift, J. Operator Theory 51 (2004) 181-200.

[10] Conway JB, Ptak M, The harmonic functional calculus and hyperreflexivity, Pacific J. Math. 204 (2002) 19-29.

[11] Duren P, Schuster A, Bergman spaces, Mathematical Surveys and Monograps 100, The American Mathematical Society, Rhode Island, 2004.

[12] Giselsson O, Olofsson A, On some Bergman shift operators, Complex Anal. Oper. Theory 6 (2012) 829-842.

[13] Hedenmalm H, Jakobsson S, Shimorin S, A biharmonic maximum principle for hyperbolic surfaces, J. Reine Angew. Math 550 (2002) 25-75.

[14] Hedenmalm H, Korenblum B, Zhu K, Theory of Bergman spaces, Graduate Texts in Mathematics 199, Springer-Verlag, New York, 2000.

[15] Louhichi I, Olofsson A, Characterizations of Bergman space Toeplitz operators with harmonic symbols, J. Reine Angew. Math. 617 (2008) 1-26.

[16] McCullough S, Richter S, Bergman-type reproducing kernels, contractive divisors, and dilations, J. Funct. Anal. 190 (2002) 447-480.

[17] Olofsson A, Wandering subspace theorems, Integral Equations Operator Theory 51 (2005) 395-409.

[18] Olofsson A, A characteristic operator function for the class of $n$-hypercontractions, J. Funct. Anal. 236 (2006) 517-545.

[19] Rudin W, Principles of mathematical analysis, third edition, McGraw-Hill, 1976.

[20] Rudin W, Real and complex analysis, third edition, McGraw-Hill, 1986.

[21] Shimorin S, Wold-type decompositions and wandering subspaces for operators close to isometries, J. Reine Angew. Math. 531 (2001) 147-189.

[22] Shimorin S, An integral formula for weighted Bergman reproducing kernels, Complex Var. Theory Appl. 47 (2002) 1015-1028. 
[23] Shimorin S, On Beurling-type theorems in weighted $\ell^{2}$ and Bergman spaces, Proc. Amer. Soc. 131 (2003) 1777-1787.

[24] Sz.-Nagy B, Foias C, Harmonic analysis of operators on Hilbert space, North-Holland, 1970.

[25] Zhu K, Operator theory in function spaces, Marcel Dekker, 1990.

Mathematics, Faculty of Science, Centre for Mathematical Sciences, Lund UniverSity, P.O. Box 118, SE-221 00 Lund, Sweden

E-mail address: olofsson@maths.1th.se and wennman@maths.1th.se 